\title{
Blended Learning and Data Use in Three Technology-Infused Charter Schools
}

\author{
Ellen B. Mandinach and Ryan C. Miskell
}

\begin{abstract}
This study examined how the affordances of technologies in the blended learning affected teaching and learning activities. The study used mixed methods to examine whether the blended learning environments provided enhanced access to and more diverse data for teachers and students from which to make educational decisions. The study found that the technologies provided more diverse data to administrators, teachers, and students and allowed for flexible adaptations to virtual and face-to-face learning to meet students' needs. The blended environments helped to create data cultures within the schools where educators used data to communicate and have an impact on instructional activities.
\end{abstract}

\section{Background}

This paper explores how the affordances of blended learning environments provide data for educational decision making to educators and students that might not be otherwise available. Because blended environments have significant technology infusion, technologies are likely to yield data that can assist educators to inform their practice in formats and real-time feedback loops not possible in more traditional settings, while also engaging students to examine their own data. Blended environments may alter the structure of classrooms by changing how instructional activities are delivered and by making the role of students more active in the teaching and learning process.

Blended learning has become an emphasis for policymakers. It is seen as the customization of educational and instructional activities to address individual students' needs. Yet Cavanagh (2014) notes, there still is no common definition. The Data Quality Campaign (2016) noted the importance of blended environments, the technology, and the resulting data, to customize instruction to meet the needs of all students (Guidera, 2016; Holiday, 2016; Messer \& Polis, 2016; Perdue, 2016). Such environments provide for real-time data, teacher and student empowerment, immediate course corrections, the use of data to individualize for every student, and connectivity to reach all students regardless of circumstances.

Research on blended learning has begun to emerge. In particular, the research reported here is informed by the work of Pane, Steiner, Baird, and Hamilton (2015). Using a multi-method approach, the RAND research identified five domains and 13 attributes of blended learning: 
- Learner Profiles

- Personalized goals for students

- Student data provided and discussed with students

- Personal Learning Path

- Outside of school learning

- Flexible/multiple paths for students through content

- Individual student support

- Competency-Based Progression

- Student progress through content based on competency

- On-demand assessment to demonstrate competency

- Flexible Learning Environment

○ Student grouping

- Learning space supports model

- Structure of learning time

- Extended learning time for students (extended school day or year)

- Technology available to all students

- College and Career Readiness

- Developing college/career preparation skills

\section{Affordances}

Achieving the aims of blended learning is a formidable goal. To do so, iNACOL (Patrick, Kennedy, \& Powell, 2013) posits several affordances that should be incorporated into blended learning models.

Highly personalized, customizable, and student-centered. The learning environment provides students some control over how they learn. Instruction is differentiated, pacing is flexible, and interventions and supports for students are available on demand. Learning environments should respond to student needs and interests, be developmentally appropriate, and be informed by data and based on the science of teaching and learning.

Competency-based and rigorous. Learning objectives are explicit, measurable, and align with college and career readiness standards. Students should be able to demonstrate their mastery at any time, without having to adhere to a rigid instructional pacing schedule.

Data-driven. Instruction to facilitate student learning should be informed by frequent and varying forms of meaningful formative and summative assessments. These data can be delivered in real time. Additionally, other sources of diverse data are made possible. An essential component here is that the technologies provide both teachers and students access to a wide variety of data made possible by technology. 
Equitable and accessible. Students are afforded access to courses, experiences, and teachers that they otherwise would not have (e.g., remote communication) as well as multiple methods of instruction and digital content (Aspen Institute Task Force on Learning and the Internet, 2014; Fernandez, Ferdig, Thompson, Schottke, \& Black, 2016).

Staffed "flexibly" but capably. Flexible staffing permits instruction and learning to happen any time.

The possibilities afforded by blended learning are stimulating growing interest in effectively using technology to support appropriate teaching, learning, and assessment environments. With sophisticated tools, educators can harness information from a wide variety of sources, including from the "digital ocean" (DiCerbo \& Behrens, 2014), to achieve the goals of blended learning (Bienkowski, 2014; Bienkowski, Feng, \& Means, 2012). For example, innovations in flexible learning technology aspire to incorporate information about individual students such as prior academics, prior and real-time cognition, on-task and perseverant behaviors, among other data to adjust digital learning environments for individual students (Abell, 2006; Fletcher, Scaffhauser, \& Levin, 2012).

There are several key advantages here. First, teachers and students save time through access to data and resources that may or may not be available without the technology in a cost-effective manner. Second, the diversity of data provides teachers and students with data that might not be available in traditional classrooms. Third, the data arrive in real time, providing tighter feedback loops among instruction, assessment, data collection, analyses, and interpretation. This iterative cycle means that data collection and feedback are much more tightly woven to the teaching and learning process.

\section{Trends}

Although the history around blended learning is not particularly long, it is possible to discern patterns and trends that are emerging. DreamBox (2014) identified 10 trends:

- Deeply student-centered learning experiences;

- Soaring numbers of digital learners;

- Supporting standards and higher-order thinking skills;

- Realizing benefits for students and teachers;

- Data-driven instruction to personalize learning;

- Personalized learning accompanied by a blended iterative approach;

- Productive gamification;

- The mobile world is where learners live now;

- Bring Your Own Device (BYOD) capacity; and

- More broadband. (p. 3)

Some of these trends are relevant to the work described here. For example, creating a student-centered environment requires active engagement on the part of learners. Similarly, a focus on higher-order skills requires students to engage in deeper cognitive activities with more creativity and less passive 
learning. Engagement affects not just students, but also changes the role of teachers. Blended environments tend to be more active, in classrooms and with virtual connections. They also provide data that heretofore might not be readily available in real time that allow for customizable teaching and learning experiences. Finally, with the proliferation of mobile devices, emerging technologies, and more ubiquitous connectivity, blended environments provide for more flexibility throughout the teaching and learning process.

When the U. S. Department of Education (2016) released its most recent national education technology plan, blended learning loomed large. The plan emphasizes the role of technology in the design of teaching and learning activities and notes that:

Teachers collaborate to make instructional decisions based on a diverse data set, including student and teacher observations and reflections, student work, formative and summative assessment results, and data from analytics embedded within learning activities and software aided by real-time availability of data and visualizations, such as information dashboards. (p. 41)

The plan also emphasizes the role of technology for assessment, providing new and more innovative means by which to measure learning. The plan lays out that the next generation of assessments will be: embedded in learning, adaptive to learners' ability and knowledge, with feedback given in real time, and with enhanced item types that can measure complex competencies. Whether through assessment platforms or data dashboards, such data have the potential to provide more meaningful and timely information to students and teachers about learning progressions. Data will be more diverse, in-depth, and informative for teaching and learning. In combination, the diverse data and the immediacy of feedback will enable more highly adaptive learning experiences made possible by the technology.

Recognizing that the current state of data systems makes interoperability a challenging prospect at best, the plan stresses the need to overcome this issue so that data from multiple sources, including formative and summative assessments, can reside in one repository. The single silo will facilitate easier access for teachers, students, data teams, and administrators. It will enable the triangulation among diverse data sources to obtain more comprehensive pictures of students.

Having the technology infrastructure in place is important, but so is the provision for the human infrastructure; that is, educators must know how to use the data effectively and responsibly (Data Quality Campaign, 2014; Mandinach \& Gummer, 2016). They must be data literate. Having students also involved in the examination of data also is a recommendation posed by the Practice Guide on data-driven decision making released by the Institute of Education Sciences (Hamilton et al., 2009). 


\section{Methods}

This study used a purposive sample of three charter schools, two high schools and one middle school, serving socioeconomically challenged students. The schools were targeted because of their history of infusing diverse technologies into their educational solutions. The schools also have strong building leadership focused on the use of technology and data to support teaching and learning, reflecting the recommendations from the IES practice guide (Hamilton et al., 2009).

The study focused on a core sample of 15 teachers. The study also included building administrators who provided leadership in terms of technology infusion, data use, professional development, and a commitment to enhance teaching and learning.

The study used multiple measures to triangulate data sources. Measures included interviews with teachers and administrators, a survey, multiple classroom observations, an artifact protocol, and a data literacy protocol. Each of these measures focused on the collection of data to address the following:

- What are the technological applications used in the classrooms, schools, and through mobile technologies to enhance teaching and learning?

- What are the affordances of these technologies that provide data to teachers and students to enhance teaching and learning? Are these data unique to the technological applications or are they readily available without the technology?

- What other resources or artifacts do teachers use to enhance their classroom practices?

- What are the supportive resources provided by administrators to make possible the blended learning environments?

Data were collected over a two-year period with several visits to the schools. During that time, the schools experienced some turnover in the teaching staffs, so additional teachers were added to the sample.

\section{Results}

Results from the multiple sources of data collection are reported based on the specific method.

\section{Interviews}

Interviews were conducted with all participating teachers and administrators from the three schools during the first site visit. As new teachers joined the sample, interviews were conducted with them. A second set of interviews was conducted to discuss progress and changes over the course of the two academic years. 
Administrator interviews. The students served by the schools are primarily Hispanic and African-American. Most students come from impoverished homes. Some students are in group or custodial homes. Over 90 percent are on free or reduced lunch. Many students have attendance issues due to long commutes. The students may also lack traditional, supportive home environments where parents or guardians can take an active role in the education process. Because the students face many environmental challenges, the explicit vision for all educators is respect for student needs. Educators hold high expectations for all students and strive to have them attain college. They do this through a nurturing and flexible environment that attempts to customize to the students' evolving needs. Technology plays a major role.

Technology integration is a top priority. The use of technology is seen as a way to deal with relevance because the students have all grown up with technology. They use technology to supplement and enhance instruction, not supplant it. Educators use various technologies to enable students to be connected from anywhere at any time. The technologies are diverse. All classrooms have Smartboards, projectors, COWs (computers on wheels: 30 notebooks), laptops, responders/clickers, graphing calculators, and smart tablets. They use some of the state technology, including connections to data tools and dashboards, to provide a different level of data. They use the Galileo assessment system to provide formative assessments and to engage students in data conversations. Students are expected to accept responsibility for their own learning. Students are also expected to plot their own data and monitor their progress, understanding what they need to do to improve. The philosophy is that data drive the discussions.

The administrators provide many resources for teacher professional development around instruction, curricula, data, and technology. They bring in professional development providers on a regular basis, send teachers to conferences, and provide professional development on Wednesday afternoons, where technology and data use are expected.

The schools have a robust data collection system and process. There is a center server and data system, yet there are still data silos given the diversity of technological applications. The principals are data savvy and constantly working to improve the technology and human capacity of their staffs. There is one central "data guy" that serves all three schools. Diverse data are collected, not just student performance: family contact information, medical (vaccination, special needs), attendance, tardiness, early dismissal, transcripts, missing assignments, grades, languages, family circumstances, growth reports, and other data. There is a FamilyLink for families and students to gain access to the system.

Teacher interviews. The teacher interviews yielded information about four general topics: teacher beliefs around technology and data use; components of blended learning environments; technology; and data use. All of the teachers reported that they believed that using data has the potential to improve instructional practice and help to meet their students' needs. Twelve teachers (80\%) mentioned that the use of data helps them to consider what to teach, what to reteach, and allows them to plan their instruction. Thirteen teachers (87\%) commented that data drive decisions and their practice must be grounded in data. Data tell the teachers where to go and what gaps need to be addressed. The take-away message was that the teachers could not meet the needs of the students without data and the technologies made possible teacher and student data use. 
Every teacher noted that there was an explicit vision for the use of technology. The vision included innovation, meeting the needs of diverse learning through many media, getting technology in the hands of teachers and students to enhance the teaching and learning process, and providing a supportive environment where everything is data driven. The foundational mission is to help all students to live to capacity and succeed.

Teachers were asked how their blended classrooms differ from more traditional ones and what the value-added is from these environments. All of the teachers mentioned that blended learning creates an environment that is more active and engaging for students and requires more hands-on activities. The environment is less formal because it lends itself to more project-based and learner-centered activities. All teachers noted that the blended environment gives students access to a digital world to which they might not otherwise have access. It makes them digital citizens. It therefore has the capacity to level the playing field and provide a door to learning for even the most challenged students.

For teachers, the blended classroom provides significant time savings through the learning technologies, particularly the Galileo assessment system. Galileo provides immediate feedback to teachers and students that would not be possible without the technology, particularly in terms of the drill-down capacity for item analyses. It allows both teachers and students to know where students are in relation to state standards and helps the teachers to formulate and plan instruction.

The blended environment provides a great deal of flexibility in how to provide instruction. There can be individualization with a total customization to each student's needs. There can be small groups or full class instruction. The technologies allow teachers to know where each and all students are in terms of learning goals at all times. Student learning and growth can be monitored at global or fine-grained levels. This flexibility enables teachers to pinpoint students' strengths, weaknesses, and learning needs. The blended environment stimulates better organizational skills and efficiency for both teachers and students through access to diverse data sources that inform the teaching and learning process. Regardless of discipline, the data provided by the technologies enable teachers and students to gauge learning progress through rapid or immediate feedback. The environment promotes the use of diverse data because of the affordances of the technologies that might not be possible in more traditional educational settings. The affordances relate not only to the types of data, but also the feedback loop from which teachers and students are able to access the data. The performance-based data help to guide instruction and allow teachers to know what to reteach.

A component of blended environments is the changing role of the student. These environments place more responsibility on students for their own learning and monitoring their progress through self-examination of data. Essentially, students must become their own data-driven decision makers. Students must self-monitor. They look at their data and monitor their own performance. Because of this, students are more active, engaged, and motivated. They must take more responsibility and ownership for and pride in their own learning. Teachers report that students grow to understand expectations by monitoring their progress. They gain time management skills and collaborative skills. 
Technologies are a major component in blended environments, yet they presented some challenges. The multitude of technologies made possible the blending of instruction and learning. WiFi was essential yet somewhat problematic. WiFi was readily available, although bandwidth was insufficient for the demand. Another challenge to having so many technologies is the fact that they often work in isolation with limited interoperability. Indeed, this was the case across the three schools. Applications worked in isolation. Connections were rare and data sharing pretty much nonexistent.

Teachers were asked about their access to data and the sources of data they use. They all reported that they had access to many sources of data. Most of the teachers (93\%) focused on the data from the Galileo assessment system, noting that without the system and its analytics, the data process would be impossible and too labor intensive. All teachers were clear that they used much more than assessment and student performance data. Some teachers (33\%) mentioned motivational, perception, attendance, behavioral, observational, attitudinal (mood), physical, medical, group processes, background (family), demographic, and coping strategy data. It was clear, though, that the data from Galileo, and the system's ability to drill down to item analyses, loomed large as a data source. Because of the frequent conflation between data literacy and assessment literacy (Mandinach \& Gummer, 2016), teachers were asked specifically if data are only about assessments or data are broader. All teachers acknowledged the centrality of assessment data, but also the need to consider the whole child, context, and background.

Yet even with the plethora of data available to them, the teachers acknowledged that there were data they would like to have. Many teachers wanted historical or longitudinal data. They wanted more data on family issues and background (graduation history), arrest records, and background or contextual data to help them gain a more holistic understanding of their students.

\section{Artifact Protocol}

The Artifact Protocol was given to all teachers during their initial interview. It asked the teachers to identify the data they use in the course of their practice. Results reflect data from 14 teachers. Teachers reported that they use diverse materials and resources. These include research (10), public media (11), textbooks (9), and diagnostic materials (11). All but one teacher reported using a variety of websites. They consulted websites for their specific disciplines, assessment websites, social media, and professional websites. All respondents reported that they sought help from and consulted colleagues and other professionals. These included administrators, other teachers, the school "data guy," supervisors, former professors, formative assessment professionals, colleagues at conferences, disciplinary specialists, and state data specialists.

\section{Classroom Observations}

Classroom observations were conducted for all participating teachers. Some teachers were observed multiple times. The observations noted the classroom setup, forms of technologies in the classroom, media and resources use, type of classroom activities, number, composition, and grouping of students, and structure of the classroom. 
Technology loomed large in all classrooms. Every room was outfitted with a SmartBoard, projection system, computers, laptops, and tablets. Other technology applications were used as needed, depending upon the discipline. All classrooms contain high-bandwidth WiFi.

Classrooms in the two high schools were set up around student groups to maximize student interactions. Seating was in pods of students. These classrooms were centered around the use of the SmartBoards. In contrast, the classrooms in the middle school were set up more traditionally, in rows to accommodate the larger number of students. These classrooms also focused on the SmartBoards, but also contained "COWs." Each classroom had a large mobile container on wheels from which teachers would extract laptops or tablets to distribute to the students for personalized work.

Classroom observations yielded rich examples of how different teachers took advantage of the technologies to enhance the teaching and learning process.

- A high school math teacher taught a lesson on seriation. Students sat in small groups with tablets to work on the lesson. The teacher used the SmartBoard where students presented their work and defended their responses to the problem.

- A student approached a high school math teacher with his mobile device. He proudly announced that he had worked through 80 percent of the online assessment remotely. The teacher had set a deadline for completion of the unit and the student remotely worked to meet the personalized time line for mastery of the materials.

- A high school science teacher mixed instructional approaches on a biology topic. She provided selected video clips. She called individual students to her computer to review their progress. During this review, she retrieved each student's electronic portfolio to discuss what they had done well and topics that still needed attention.

- A middle school social studies teacher conducted a lesson on income disparities in Middle Eastern countries. She provided a structured worksheet with questions to facilitate students' online research. The students accessed media from the specific countries to research topics such as average income and gender income disparity. The teacher then asked the class about their findings and the sources of the information, then engaged them in a discussion about income in the countries compared to similar data from Arizona and the United States.

- A high school English teacher worked with her class on an exercise on advertising. Students were grouped together, using tablets, to examine advertisements around which structured questions helped the students conduct their research. The teacher moved among the groups, stimulating discussion about their findings. She then brought the class together for a full-class discussion and presentation of group findings.

- A middle school physical education teacher covered a unit on nutrition and fitness. Students accessed a website that presented the key principles of good nutrition and fitness. Students downloaded an app to calculate their activity relative to the principles. They charted their eating and exercise patterns over time and related this to the key principles. 
Observations indicate that the affordances of the technologies were being used creatively and effectively to meet the individual needs of students and to customize instruction in ways to facilitate student learning. That said, there were observations where the classrooms looked no different than more traditional ones. For the most part, however, the teachers used the technologies for planning, instruction, and assessment. Students used the mobile devices for remote learning and assessment, and for activities

in the classroom. Teachers were able to monitor student performance and discuss results with the students to keep them on pace. Students were engaged in the performance conversations. Data were readily available to teachers and students that might have been impossible or impractical without the technologies.

\section{Data Literacy Protocol}

The Data Literacy Protocol was developed to capture the skills of the data literacy for teachers (DLFT) construct (Gummer \& Mandinach, 2015). Given the construct, it was expected that some of these skills would be manifested in classroom practice and observable through classroom observations, whereas others might be exhibited during data-teaming activities, or outside of the classroom. Data were collected for 23 classroom observations, seven of which were repeated measures to determine consistency within a teacher.

The range of observed skills was from 2 (determine unintended consequences) to 23 (monitor student progress). The most readily observable skills were those that affect instructional practice. The most frequently observed skills included individualizing instruction, planning classroom practice, designing classroom practice, and implementing classroom practice (22 teachers). Almost all the teachers showed evidence of articulating a problem of practice (22), monitoring classroom practice shifts (22), using formative and summative assessments (21), adapting current classroom practice based on immediate student feedback (21), incorporating student work as a data source (20), recognizing and using informal classroom information as a source of data (20), adjusting classroom practice (20), and engaging students with their personal results (20).

The least frequent skills were those that are not readily observable in classroom practice. They include: the ethical use of data (3); belief in data (4); comparing data pre- and post-decision (4); probing for causality (4); identifying inaccurate, misleading, or out-of-range data (4); prioritizing data (4); understanding basic statistics such as measures of central tendency and dispersion (5); using statistics (5); reanalyzing the original problem or decision (5); testing hypotheses (5); and considering the need for an iterative decision cycle (5).

\section{Survey Results}

Teachers were asked to complete a survey during the first year of data collection. The survey covered several topics related to school culture, technology, data, and leadership. Fifteen teachers completed the survey. 
Teachers held favorable views of their colleagues' efforts to support one another and focus on student learning. Respondents agreed that colleagues collaborate $(12,80 \%)$ and are focused on the mission of improving student learning (13,87\%). Thirteen respondents agreed that administrators at the school are supportive of teachers, and trust teachers to make decisions about their own instruction.

Teachers identified a number of school conditions that serve as obstacles to their efforts to promote student learning using technology. Nine teachers (60\%) believed that students' inadequate technology skills are an obstacle. Six teachers $(40 \%)$ believed the lack of technical support is an obstacle to student learning. Six teachers (40\%) reported there were too few computers and devices to accommodate all students. Eleven respondents (73\%) believed that slow WiFi and/or inadequate bandwidth are obstacles. Nine respondents $(60 \%)$ reported that hardware problems have been obstacles to their efforts to promote student learning.

Accountability loomed large. Eleven respondents (73\%) faced pressure as a result of state or district standards and/or testing requirements to cover specific content. This pressure was an obstacle to their efforts to promote personalized learning. Eleven respondents (73\%) reported they spend excessive time developing personalized content for students. Seven teachers $(47 \%)$ said they have inadequate data to personalize instruction.

Absenteeism was a major issue. All teachers reported high levels of absenteeism as an obstacle to their efforts to promote personalized learning. Discipline also was an issue. Twelve teachers (80\%) believed that student disciplinary problems present obstacles to personalized learning.

Student performance data are the major data source for teachers, whether in traditional or blended environments. Teachers reported the use of student achievement data to: tailor instructional content and pace to student needs (10); develop recommendations for tutoring and support services (13); identify topics requiring more or less emphasis in their instruction (14); reflect on and discuss teaching and learning with their colleagues (13); and discuss learning with their students (13).

Not all data that teachers used are achievement-oriented. Many other data sources existed. In the 2014-15 school year, five teachers (33\%) reported using nonachievement outcomes (e.g., student behavior and attitudes). Of these five teachers (33\%), four (27\%) used the data to tailor instruction to individual students' needs; three (20\%) to tailor the content of instruction to individual students' needs; all five to develop recommendations for tutoring or other support services for particular students; and three (20\%) to reflect on and discuss learning with their students.

Teachers had favorable views of their school's data system. Ten teachers (67\%) believed they have access to high-quality assessment data that help them adapt the pace or content of instruction. Ten teachers $(67 \%)$ reported the data system provides real-time data that are actionable. Ten teachers $(67 \%)$ believed the data system provides information at a level of detail that helps them inform their instruction. Eight teachers (53\%) believed they have sufficient data, but need help to translate data into actionable steps. 
The survey asked about usage patterns and the sources of data that teachers access. Data closer to the individual student and classroom level (e.g., attendance and personal data) were used more frequently by teachers than other forms of data (e.g., state data).

The role of building leadership in data use is well established (Hamilton et al., 2009). Leadership in the schools confirmed that finding. All 15 teachers reported that administrators encourage data use to support effective teaching. Fourteen teachers (93\%) believed the administrators create opportunities for teachers to use data. Twelve teachers ( $80 \%$ ) believed the administrators ensured teachers have training for data use. All teachers reported the administrators discussed data with them. Fourteen teachers (93\%) stated the administrators created protected time for using data.

Teachers agreed that technologies are useful. All 15 teachers believed they have the proper technology to efficiently examine data. Fourteen teachers (93\%) reported the computer systems provide them with access to lots of data. Twelve teachers ( $80 \%$ ) believed the computer systems allow them to examine various types of data and $13(87 \%)$ reported the computer systems generate displays and reports that are useful. Generally, teachers reported the available technologies to be useful in their classrooms. Eight teachers (53\%) reported the COWs to be useful. Five teachers reported the tablets useful. Twelve teachers $(80 \%)$ said the Smartboards were useful. Twelve teachers $(80 \%)$ reported the WiFi to be useful. Ten teachers $(67 \%)$ reported that mobile devices were useful in the classroom.

\section{Discussion}

It is clear from the results that teachers used the richly infused technology and the resulting sources of data to reach their students. This might not be done as effectively without the affordances provided by the technologies. Teachers and students had anytime and anywhere access that allowed them to maximize learning opportunities. Teachers had in-depth item analyses linked to state standards to help them monitor student progress and plan instruction. Teachers reported that the blended environment changes the functioning of the classroom and the role of the student. Teachers also reported that students were more active, engaged, motivated, and involved in their own learning.

All 13 blended components from the RAND work (Pane et al., 2015) were observed in the schools. The schools used personalized goals to structure instructional plans for each student. Educators used student data to inform instruction and involved students in data-based discussions. Teachers used diverse data and resources to meet the student needs. Data were real time, plentiful, and available. Technologies provided anytime and anywhere access to outside of school learning opportunities. The schools provided out-of-school learning opportunities through access to the technological platforms. Teachers created flexible and multiple paths for students through content materials by customizing instruction to individual student needs. Students worked at their own pace. They also provided individualized student support. Galileo helped teachers ensure that students progressed through the content based on a competency model. Galileo also provided on-demand assessments through which students were able to demonstrate competency, and provided teachers with real-time data that could immediately inform instructional 
planning. Teachers used different grouping strategies in flexible ways. Classrooms provided learning spaces that supported the technologies and the diversity of learning objectives. Teachers used the affordances of the technologies to provide flexible learning time and extended learning time beyond the traditional school hours to meet the students' transportation schedules. Teachers stayed late to work with students and provided communication strategies after school hours to meet student needs. Technology was made available to all students. In class, each student had a dedicated laptop or tablet. Finally, the two high schools provided students with life skills and college and career preparedness. One school's vision included an explicit goal to prepare its students for college. Another school focused on career-ready skills through courses offered by the career and technical education teacher, whose courses emphasized job readiness.

Taking the RAND report's 13 attributes at face value, the schools exhibited the components identified as important to blended learning environments. It is important to note, though, that these schools have characteristics that may not be readily generalizable. First, they were infused with many different technologies and well-trained teachers. Second, one of the technologies (Galileo) provided a rich source of real-time, student performance data that helped teachers make rapid turnaround instructional decisions and modifications. Third, leadership of the schools had explicit visions for student performance, using the technologies and data to undergird all teaching and learning activities as well as administrative decision making. These schools had strong leaders that provided a conducive and open context that promotes collaboration, respect, and trust among the educators. Fourth, the educators recognized the importance of how the blended environments provided flexible and customizable contexts to meet the many challenges of their student populations. The technologies provided the anytime and anywhere extension beyond the brick and mortar of the school buildings. Given the high absenteeism, mobile devices allowed students to use technology in their own time and space. The only limitation was that some students had to seek WiFi hubs because the devices lacked data plans.

In reviewing the school characteristics, it is important to note that the findings are also aligned to the five recommendations of the IES practice guide on data use (Hamilton et al., 2009). The first recommendation pertains to using an ongoing inquiry cycle to inform instruction. Clearly, the staff of these schools were identifying problems of practice, collecting data, and making decisions based on those data to inform their practice. The second recommendation is to involve students in the examination of their own data. Students in the schools were taking an active role, being their own data-driven decision makers. Teachers worked with them to set expectations and learning goals, and use data to monitor their progress. The third recommendation is to establish a vision for data use. As noted above, the administrators explicitly led with data. Their visions were well articulated and modeled. The fourth recommendation is to create a culture of data use. This means providing the necessary supports and resources to establish a data culture. The schools created data teams with dedicated meeting times. There was a "data guy" to help the schools with data-related issues. Administrators made possible the time and resources for professional development, technical assistance, and attendance at relevant conferences. Finally, the fifth recommendation is to have a data system. The district had a central data system, but also used other siloed applications that served as repositories for data. Similar to the findings in other case studies 
(e.g., Bernatek, Cohen, Hanlon, \& Wilka, 2012a, 2012b, 2012c), the data systems lacked interoperability and data sharing capacity, leaving each system to function in isolation. This is a major issue due to the proliferation of diverse sources of data.

Other challenges existed such as data sharing, interoperability, and the plethora of and accessibility to data that were generated through blended learning. With the proliferation of data from diverse sources come concerns about the protection of data privacy. The collection and examination of these data sources, particularly in real time, provide great opportunities for the customization of teaching and learning. Yet, even greater challenges exist in terms of maintaining the safety and security of the data to protect students' privacy and confidentiality (Data Quality Campaign, 2016; Herold, 2014) and ensure that the educators are knowledgeable about how to use data not just effectively, but responsibly (Mandinach \& Gummer, 2016). Data privacy has become a key issue and continues to be a concern.

Blended learning certainly has taken hold in these three schools. These are charter schools serving a relatively small number of students. The question of the model's generalizability is of concern. More traditional schools are not likely to have the similar resources. They may have less technology. They may lack a vision for the use of technology and data. They may not have an embedded data culture and technology that extends within and beyond the school boundaries. They may not be provided with the extensive learning opportunities found in these schools in terms of professional development, technical assistance, and conference attendance. That said, there is much that can be learned from these three schools about how the alignment of RAND's 13 attributes (Pane et al., 2015) and the IES practice guide's five recommendations (Hamilton et al., 2009) in school settings can create blended learning environments that have the potential to reach even the most challenged students and help them to succeed.

\section{Acknowledgments}

This study was funded by a grant from the Bill and Melinda Gates Foundation to WestEd. The results and opinions are those of the authors. The authors would like to acknowledge the willingness of the educators in the three Arizona schools to participate in the study.

\section{References}

Abell, M. (2006). Individualizing learning using intelligent technology and universally designed curriculum. Journal of Technology, Learning, and Assessment, 5, 21.

Aspen Institute Task Force on Learning and the Internet. (2014). Learner at the center of a networked world. Washington, DC: The Aspen Institute. Retrieved from http://csreports.aspeninstitute.org/documents/AspenReportFinalPagesRev.pdf

Bernatek, B., Cohen, J., Hanlon, J., \& Wilka, M. (2012a). Blended learning in practice: Case studies from leading schools: KIPP. Austin, TX: Michael \& Susan Dell Foundation. Retrieved from http://www.fsg.org/publications/blended-learning-practice 
Bernatek, B., Cohen, J., Hanlon, J., \& Wilka, M. (2012b). Blended learning in practice: Case studies from leading schools: Rocketship Education. Austin, TX: Michael \& Susan Dell Foundation. Retrieved from http://www.fsg.org/publications/blended-learning-practice

Bernatek, B., Cohen, J., Hanlon, J., \& Wilka, M. (2012c). Blended learning in practice: Introduction to case studies from leading schools. Austin, TX: Michael \& Susan Dell Foundation. Retrieved from http://www.fsg.org/publications/blended-learning-practice

Bienkowski, M. (2014, April 6). Putting the learner at the center: Exposing analytics to learning participants. Paper presented at the annual conference of the American Educational Research Association, Philadelphia, PA.

Bienkowski, M., Feng, M., \& Means, B. (2012). Enhancing teaching and learning through educational data mining and learning analytics: An issue brief. Washington, DC: U.S. Department of Education. Retrieved from http://www.ed.gov/edblogs/technology/files/2012/03/edm-la-brief.pdf

Cavanagh, S. (2014, October 22). 'Personalized learning' eludes easy definitions. Education Week: A Special Report on Personalized Learning, 34(9), S2-S4.

Data Quality Campaign. (2014, February). Teacher data literacy: It's about time: A brief for state policymakers. Washington, DC: Author.

Data Quality Campaign. (2016, April). Time to act: Making data work for students: National summit. Washington, DC: Author.

DiCerbo, K. E., \& Behrens, J. T. (2014). Impacts of the digital ocean on education. London: Pearson. Paper presented at the annual conference of the American Educational Research Association, Philadelphia, PA.

DreamBox Learning. (2014). Blended learning innovations: 10 major trends. Retrieved from http://www.dreambox.com/white-papers/blended-learning-innovations-10-major-trends

Fernandez, H., Ferdig, R. E., Thompson, L. A., Schottke, K., \& Black, E. W. (2016). Students with special health care needs in K-12 virtual schools. Educational Technology \& Society, 19, 67-75.

Fletcher, G., Scaffhauser, D., \& Levin, D. (2012). Out of print: Reimagining the K-12 textbook in a digital age. Washington, DC: State Technology Directors Association.

Guidera, A. R. (2016, April). It's time to act. Speech given at the Data Quality Campaign's National Summit, Time to Act: Making Data Work for Students, Washington, DC.

Gummer, E. S., \& Mandinach, E. B. (2015). Building a conceptual framework for data literacy. Teachers College Record, 117(4). Retrieved from http://www.tcrecord.org/PrintContent.asp?ContentID=17856

Hamilton, L., Halverson, R., Jackson, S., Mandinach, E., Supovitz, J., \& Wayman, J. (2009). Using student achievement data to support instructional decision making (NCEE 2009-4067). Washington, DC: National Center for Education Evaluation and Regional Assistance, Institute of Education Sciences, U.S. Department of Education. Retrieved from http://ies.ed.gov/ncee/wwc/publications/practiceguides/.

Herold, B. (2014, October 22). Balancing privacy and innovation. Education Week: A Special Report on Personalized Learning, 34(9), S8, S11.

Holiday, T. (2016, April). From priority to action: Make this vision a reality. Speech given at the Data Quality Campaign's National Summit, Time to Act: Making Data Work for Students, Washington, DC. 
Mandinach, E. B., \& Gummer, E. S. (2016). Data literacy for educators: Making it count in teacher preparation and practice. New York, NY: Teachers College Press.

Messer, L., \& Polis, J. (2016, April). Build a supportive federal framework for student success. Speech given at the Data Quality Campaign's National Summit, Time to Act: Making Data Work for Students, Washington, DC.

Pane, J. F., Steiner, E. D., Baird, M. D., \& Hamilton, L. A. (2015). Continued progress: Promising evidence on personalized learning. Santa Monica, CA: RAND. Retrieved from http://www.rand.org/pubs/research_reports/RR1365.html

Patrick, S., Kennedy, K., \& Powell, A. (2013). Mean what you say: Defining and integrating personalized, blended and competency education. Vienna, VA: International Association for K-12 Online Learning. Retrieved from http://www.inacol.org/resource/mean-what-you-say-defining-and-integratingpersonalized-blended-and-competency-education/

Perdue, B. (2016, April). Make data work for students. Speech given at the Data Quality Campaign's National Summit, Time to Act: Making Data Work for Students, Washington, DC.

United States Department of Education. (2016). Future ready learning: Reimagining the role of technology in Education: 2016 national education technology plan. Washington, DC: U. S. Department of Education, Office of Educational Technology. Retrieved from http://tech.ed.gov/files/2015/12/NETP16.pdf

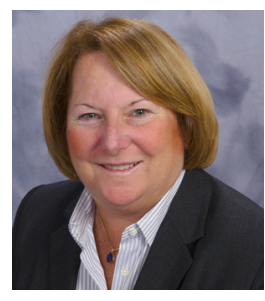

Ellen B. Mandinach is a Senior Research Scientist and the Director of the Data for Decisions Initiative at WestEd. Dr. Mandinach is a leading expert in data-driven decision making at the classroom, district, and state levels. Her work has focused on understanding how educators are using data to inform practice. Dr. Mandinach has authored many publications for academic journals, technical reports, and five books. Her most recent books are, Data Literacy for Educators: Making it Count in Teacher Preparation and Practice and Transforming Teaching and Learning Through Data-Driven Decision Making. She received a PhD in educational psychology from Stanford University.

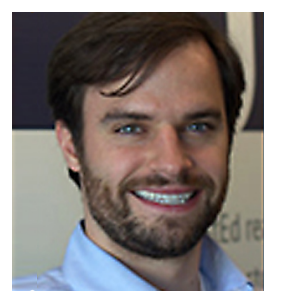

Ryan C. Miskell is a Research Associate in the Learning Innovations program at WestEd. His work involves federal, state, and district level monitoring, policy analysis, and program evaluation to inform educational policy and program decisions. Prior to joining WestEd in 2015, he was a Research Associate with the Oklahoma Center for Education Policy and a teacher with Tulsa Public Schools in Oklahoma. He received a B.A. in political science from American University, an M.Ed. in education administration, curriculum, and supervision from the University of Oklahoma, and a PhD in education leadership and policy studies from the University of Oklahoma. 